\title{
TIMs: central regulators of immune responses
}

\author{
David A. Hafler and Vijay Kuchroo
}

\begin{abstract}
Exhaustion of $\mathrm{T}$ cell responses during chronic viral infections has been observed in both mouse and man and has been attributed to up-regulation of PD-1 on the surface of exhausted T cells. In patients with chronic human HIV infection, $T$ cell exhaustion leads to opportunistic infections associated with AIDS. However, not all the exhausted T cells express PD-1, suggesting that other molecules may be involved in the phenotype. A new study now demonstrates a central role for $T$ cell immunoglobulin and mucin domain-containing protein-3 (TIM-3) in T cell exhaustion during chronic HIV infection and suggests that TIM-3 may be a novel therapeutic target in chronic viral diseases.
\end{abstract}

Efforts to generate a vaccine against HIV have been disappointing, perhaps because of the unique tropism of HIV for $\mathrm{CD}^{+}{ }^{+} \mathrm{T}$ cells. Infection with HIV is associated with rapidly acquired defects in cell cycle initiation in $\mathrm{CD}^{+}$cells and cytoxicity in $\mathrm{CD}^{+} \mathrm{T}$ cells (1). Ultimately $\mathrm{T}$ cells become dysfunctional and fail to generate immune responses, leaving the patient vulnerable to opportunistic infections. The rapid onset of $\mathrm{T}$ cell dysfunction in HIV-infected patients invariably hinders early attempts to boost the immune system using vaccines and other interventions. Exhaustion of $\mathrm{CD} 4^{+}$ and $\mathrm{CD}^{+} \mathrm{T}$ cells during HIV and other chronic viral infections has been associated with sustained expression of the inhibitory molecule PD-1, which results in inhibition of $\mathrm{T}$ cell expansion and cytokine production (2-4). However, as not all exhausted cells express PD-1, it is likely that other molecules may contribute to the exhaustion associated with HIV infection and other chronic viral infections. On p. 2763 of this issue, Jones et al. identify a novel molecular mechanism for $\mathrm{T}$ cell exhaustion mediated by the expression of TIM-3 (5).

The in TIMidation of T cells The TIM family of genes, which consists of eight members in mice and three

D.A.H. and V.K. are at Center for Neurological Diseases, Brigham and Women's Hospital, Harvard Medical School, Boston, MA 02115

CORRESPONDENCE

V.K.: vkuchroo@rics.bwh.harvard.edu members in humans (6), is located on chromosome 11 in mice and $5 \mathrm{q} 33$ in humans - genetic regions that have been associated with autoimmune and allergic diseases. TIM proteins are type 1 membrane proteins with a structurally conserved immunoglobulin variable $(\mathrm{IgV})$ domain and mucin stalk that connects to an intracellular tail. TIM proteins were initially thought to be expressed specifically on the surface of differentiated effector $\mathrm{T}$ cells and to directly regulate their activity, but we now know that TIMs are also expressed on and regulate the function of antigen-presenting cells (7). Crystal structures of the TIM molecules has revealed a unique, conserved structure with ligand-binding sites in the $\mathrm{IgV}$ domain (8-10).

TIM-3 was initially identified as a molecule expressed on T helper (Th)-1, but not Th2, cells in mice (11). Interaction between TIM-3 and its ligand galectin-9 inhibits Th1 responses (12) and induces peripheral tolerance $(13,14)$. As in mice, TIM-3 is preferentially expressed on human Th1 cells, but is also expressed constitutively on macrophages and dendritic cells (DCs) (7). Reduction of TIM-3 expression in T cells using small interfering RNA or blocking antibodies, for example, increases interferon (IFN) $\gamma$ secretion by $\mathrm{CD} 4^{+} \mathrm{T}$ cells (15), further supporting an inhibitory role of TIM-3 in human T cells.
Patient studies are consistent with this inhibitory function, as TIM-3 expression is defective on $\mathrm{CD}^{+} \mathrm{T}$ cells from patients with autoimmune diseases (15, 16). Specifically, $\mathrm{T}$ cell clones isolated from the cerebrospinal fluid of patients with multiple sclerosis (MS) express lower levels of TIM-3 and secrete higher levels of IFN $\gamma$ compared with clones isolated from healthy control subjects (15). $\mathrm{CD}^{+} \mathrm{T}$ cells from MS patients also show altered and delayed kinetics of TIM-3 upon activation, resulting in lower TIM-3 expression on circulating $\mathrm{T}$ cells and are therefore refractory to the blocking effects of TIM-3-specific antibodies (16).

TIM- 1 , by contrast, is predominantly expressed on Th2 cells and was first identified as the hepatitis A virus cellular receptor 1 (17). A unique polymorphism in the mucin domain of TIM-1 regulates hepatitis A virus infection, and also affects immune responses and resistance to asthma, allergy, and atopy (18).

\section{TIM-3 in chronic virus infection}

$\mathrm{T}$ cell exhaustion during HIV infection is accompanied by increased HIV replication and follows a characteristic pattern. $T$ cells first lose the ability to enter into cell cycle, to secrete IL-2, and to mediate cytotoxicity $(2,3)$. Over time, the exhausted cells also lose the ability to secrete other cytokines, such as IFN$\gamma$ and $\operatorname{TNF}(2,3,19)$. Initial studies suggested that $\mathrm{T}$ cell dysfunction is mediated by sustained PD-1 expression on $\mathrm{T}$ cells. Upon activation, T cells upregulates several inhibitory molecules, including PD-1, which help turn off $\mathrm{T}$ cell responses and thus inhibit $\mathrm{T}$ cell expansion.

A functional role for PD-1/PD-L1 signaling in $\mathrm{T}$ cell dysfunction was demonstrated by studies of lymphocytic 
A TIM-3 and T cell exhaustion in HIV infection

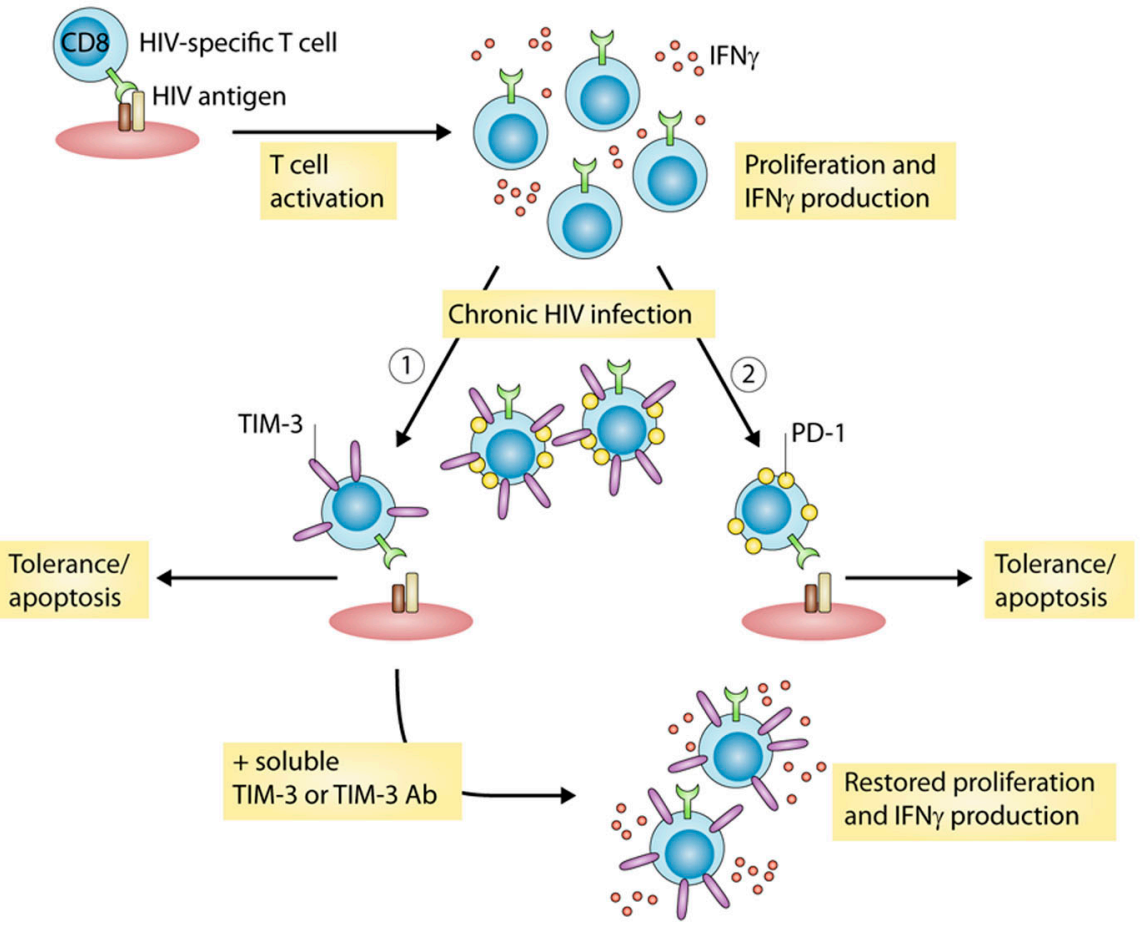

B TIM-3 and autoimmune disease

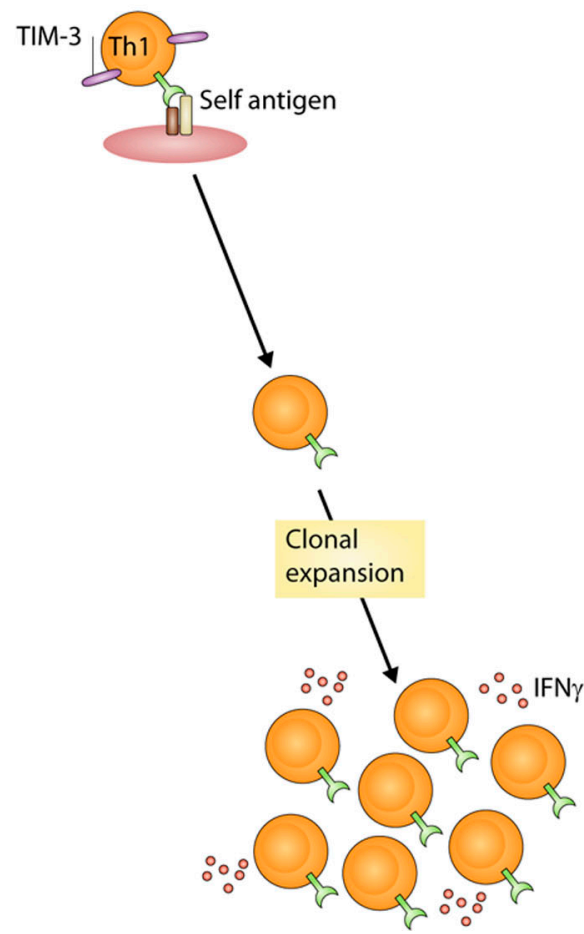

Figure 1. Differential expression of TIM-3 on the surface of T cells regulates susceptibility to viral infection or development of autoimmune injury. (A) In patients with progressive HIV infection, TIM-3 is up-regulated on the surface of CD4 and CD8 T cells. Up-regulation of TIM-3 on HIVspecific CD8 T cells leads to impaired cell survival, proliferation, and cytokine production (1). Blocking TIM-3 binding to its ligand galectin-9 using soluble TIM-3 or TIM-3 antibody restores functionality of HIV-specific CD8 T cells from HIV patients. Up-regulation of PD-1 in HIV patients also leads to T cell dysfunction (2). Although a small proportion of cells express both TIM-3 and PD-1, in the majority of cells, TIM-3 expression is distinct from PD-1, suggesting these proteins define separate populations of dysfunctional T cells in HIV patients. (B) In autoimmune diseases, autoreactive T cells upon activation and expansion down-regulate TIM-3 expression, leading to progressive uncontrolled growth and expansion of autoreactive T cells. Whether down-regulation of TIM-3 is mediated by genetic factors or by T cell activation signals in autoimmune disease is not known.

choriomeningitis virus in mice, simian immune deficiency syndrome in rhesus macaques, and HIV in humans, each of which demonstrated that blocking this pathway increased $\mathrm{T}$ cell responses, improved viral control in vivo, and enhanced the survival and proliferation of antigen-specific $\mathrm{CD}^{+} \mathrm{T}$ cells in vitro $(2,3,20,21)$. Although PD-1 expression appeared to identify a population of $\mathrm{T}$ cells entering into exhaustion, not all exhausted cells expressed PD-1 (3). Thus, the molecular pathways associated with $\mathrm{T}$ cell exhaustion, particularly in $\mathrm{CD}^{+} \mathrm{T}$ cells, remain incompletely understood.

In this issue, Jones et al. find elevated expression of TIM-3 on both $\mathrm{CD}^{+}$ and $\mathrm{CD}^{+} \mathrm{T}$ cells from individuals with acute and progressive chronic HIV infection, but not in chronically infected individuals who had controlled the infection or in uninfected individuals (5).
The frequency of TIM-3-expressing cells correlated positively with viral load and inversely with $\mathrm{CD} 4^{+} \mathrm{T}$ cell counts. These results are in sharp contrast to data showing decreased expression of TIM-3 in patients with MS. Staining with MHC class I tetramers/pentamers revealed higher TIM-3 expression on HIV-specific CD ${ }^{+}$ $\mathrm{T}$ cells than on $\mathrm{CMV}$-specific $\mathrm{CD} 8^{+} \mathrm{T}$ cells. Levels of TIM-3 expression were variable both among different individuals and among HIV-specific T cells within the same individual, suggesting that different levels of TIM-3 expression may mark cells with different functional capacities.

In a subset of patients, highly active antiretroviral therapy correlated with reduced TIM-3 expression on T cells. Sorting of TIM- $3^{+}$and TIM- $3^{-}$populations revealed that HIV-specific T cells expressing high amounts of TIM-3 secreted less IFN $\gamma$ than did TIM-3- ${ }^{-}$cell, suggesting that, like PD-1, TIM-3 may be an important marker of exhausted $T$ cells during chronic viral infections. Blocking the interaction between TIM-3 and its ligand galectin- 9 enhanced $\mathrm{CD}^{+} \mathrm{T}$ cell proliferation and cytokine production in response to HLA-A*0201-restricted HIV-1 peptides, thus solidifying the functional connection between TIM-3 expression and $\mathrm{T}$ cell dysfunction (Fig. 1). Inhibiting the TIM-3 pathway also enhanced $\mathrm{CD}^{+}$and $\mathrm{CD}^{+}$T cell proliferation when peripheral blood mononuclear cells from chronic progressors were stimulated with pooled Gag and Nef peptides. In most of the patients, TIM-3 and PD-1 were expressed on distinct populations of $\mathrm{T}$ cells, although infrequent $\mathrm{TIM}-3^{+} / \mathrm{PD}-1^{+}$cells could be detected. These data suggest that TIM-3 and PD-1 expression mark distinct subsets of exhausted $\mathrm{T}$ cells. The authors 
suggest that TIM-3 may be an important therapeutic target that is distinct from PD-1 and may help reverse exhaustion in chronic viral infections. On CD4 ${ }^{+}$cells, TIM-3 is expressed only on terminally differentiated Th1 cells. Thus TIM-3 blockade would result in the expansion of only effector $\mathrm{T}$ cells. Therapeutic blockade of PD-1, on the other hand, would expand all $\mathrm{T}$ cells, because PD-1 is up-regulated on all activated $\mathrm{T}$ cells.

\section{Open questions}

Collectively, these data suggest that TIM-3 is a central regulator of T cell responses. In autoimmunity, loss of TIM-3 function leads to excessive expansion of autoreactive effector $\mathrm{T}$ cells. In chronic HIV infection, by contrast, sustained TIM-3 expression on $\mathrm{CD}^{+}$and $\mathrm{CD} 8^{+}$ $\mathrm{T}$ cells leads to $\mathrm{T}$ cell exhaustion (Fig. 1). As with any clinical observation, these results raise many questions. It is unclear, for example, whether HIV infection itself drives TIM-3 expression or whether $\mathrm{CD}^{+} \mathrm{T}$ cells expressing high levels of TIM-3 are preferentially infected with HIV or are exhausted in their attempt to clear the virus. How the engagement of TIM-3 by its ligand galectin-9 leads to the inability of $\mathrm{T}$ cells to enter into the cell cycle and secrete cytokines is also unknown. Since TIM3-galectin-9 interactions normally induce the death of terminally differentiated Th1 cells (12), why aren't exhausted cells eliminated? And why, on the other hand, is TIM-3 expression reduced on Th1 cells in patients with autoimmune diseases such as MS?

Our recent discovery that TIM-3 is constitutively expressed by human DCs and monocytes revealed a potential role for TIM-3 in innate immune responses (7). It will be interesting to examine the function of TIM-3 on monocytes and DCs in patients with HIV infection, whether its expression changes during the course of infection, and whether TIM-3 affects the responsiveness of DCs to TLR and other innate signals.

The dysregulation of TIM-3 in patients with autoimmune disease and in chronic viral exhaustion highlight the therapeutic potential of targeting TIM3 in the treatment of these diseases. Finally, these studies highlight the utility of investigating the same regulatory pathways in patients with HIV and autoimmunity to provide unique insights into the pathogenesis of these diseases.

\section{REFERENCES}

1. Hay, C., and E. Rosenberg. 1998. Immunologic response to HIV. AIDS Clin. Care. 10: $1-3$.

2. Wherry, E.J., S.J. Ha, S.M. Kaech, W.N Haining, S. Sarkar, V. Kalia, S. Subramaniam, J.N. Blattman, D.L. Barber, and R. Ahmed. 2007. Molecular signature of CD8+ T cell exhaustion during chronic viral infection. Immunity. 27:670-684.

3. Day, C.L., et al. 2006. PD-1 expression on HIV-specific T cells is associated with T-cell exhaustion and disease progression. Nature. 443:350-354.

4. Urbani, S., B. Amadei, D. Tola, M. Massari, S. Schivazappa, G. Missale, and C. Ferrari. 2006. PD-1 expression in acute hepatitis $\mathrm{C}$ virus (HCV) infection is associated with HCV-specific CD8 exhaustion. J. Virol. 80: 11398-11403.

5. Jones, R.B., et al. 2008. Tim-3 expression defines a novel population of dysfunctional $\mathrm{T}$ cells with highly elevated frequencies in progressive HIV-1 infection. J. Exp. Med. 2763-2779.

6. Kuchroo, V.K., D.T. Umetsu, R.H. DeKruyff, and G.J. Freeman. 2003. The TIM gene family: emerging roles in immunity and disease. Nat. Rev. Immunol. 3:454-462.

7. Anderson, A.C., et al. 2007. Promotion of tissue inflammation by the immune receptor Tim-3 expressed on innate immune cells. Science. 318:1141-1143.

8. Cao, E., et al. 2007. T cell immunoglobulin mucin-3 crystal structure reveals a novel ligand binding surface. Immunity. 26:311-321.

9. Santiago, C., A. Ballesteros, L. MartinezMuñoz, M. Mellado, G.G. Kaplan, and J.M. Cassanovas. 2007. Structures of T cell immunoglobulin mucin protein 4 show a metal-ion-dependent ligand binding site where phosphatidylserine binds. Immunity. 27: 941-945.
10. Santiago, C., M. González-Freire, L. Serratosa, F.J. Morate, T. Meyer, F. Gómez-Gallego, and A. Lucia. 2007. Structures of T cell immunoglobulin mucin receptors 1 and 2 reveal mechanisms for regulation of immune responses by the TIM receptor family. Immunity. 26:299-310.

11. Monney, L., et al. 2002. Th1-specific cell surface protein Tim-3 regulates macrophage activation and severity of an autoimmune disease. Nature. 415:536-541.

12. Zhu, C., A.C. Anderson, A. Schubart, H. Xiong, J. Imitola, S.J. Khoury, X.X. Zheng, T.B. Storm, and V.K. Kuchroo. 2005. The Tim-3 ligand galectin-9 negatively regulates $\mathrm{T}$ helper type 1 immunity. Nat. Immunol. 6:1245-1252.

13. Sabatos, C.A., S. Chakravarti, E. Cha, A. Schubart, A. Sanchez-Fueyo, X.X. Zheng, G.J. Freeman, and V.K. Kuchroo. 2003. Interaction of Tim-3 and Tim-3 ligand regulates T helper type 1 responses and induction of peripheral tolerance. Nat. Immunol. 4:1102-1110.

14. Sanchez-Fueyo, A., et al. 2003. TIM-3 inhibits T helper type 1-mediated auto- and alloimmune responses and promotes immunological tolerance. Nat. Immunol. 4:1093-1101.

15. Koguchi, K., D.E. Anderson, L. Yang, K.C. O'Connor, V.K. Kuchroo, and D.A. Hafler. 2006. Dysregulated T cell expression of TIM3 in multiple sclerosis. J. Exp. Med. 203:1413-1418

16. Yang, L., D.E. Anderson, J. Kuchroo, and D.A. Hafler. 2008. Lack of TIM-3 immunoregulation in multiple sclerosis. J. Immunol. 180:4409-4414.

17. Kaplan, G., A. Totsuka, P. Thompson, T. Akatsuka, Y. Moritsugu, S.M. Feinstone. 1996 Identification of a surface glycoprotein on African green monkey kidney cells as a receptor for hepatitis A virus. EMBO J. 15:4282-4296.

18. McIntire, J.J., D.T. Umetsu, and R.H DeKruyff. 2004. TIM-1, a novel allergy and asthma susceptibility gene. Springer Semin. Immunopathol. 25:335-348.

19. Barber, D.L., E.J. Wherry, D. Masopust, B. Zhu, J.P. Allison, A.H. Sharpe, G.J. Freeman, and R. Ahmed. 2006. Restoring function in exhausted CD8 $\mathrm{T}$ cells during chronic viral infection. Nature. 439:682-687.

20. Petrovas, C., et al. 2007. SIV-specific CD8+ $\mathrm{T}$ cells express high levels of PD1 and cytokines but have impaired proliferative capacity in acute and chronic SIVmac251 infection. Blood. 110:928-936.

21. Zhang, J.Y., et al. 2007. PD-1 up-regulation is correlated with HIV-specific memory CD8+ T-cell exhaustion in typical progressors but not in long-term nonprogressors. Blood. 109:4671-4678. 